\title{
Key imaging features for differentiating cystic biliary atresia from choledochal cyst: prenatal ultrasonography and postnatal ultrasonography and MRI
}

\author{
Hyun Joo Shin ${ }^{1,2}$, Haesung Yoon ${ }^{1,2}$, Seok Joo Han ${ }^{2,3,4}$, Kyong Ihn ${ }^{2,3,4}$, Hong Koh ${ }^{2,5}$, \\ Ja-Young Kwon ${ }^{6}$, Mi-Jung Lee ${ }^{1,2}$ \\ 'Department of Radiology, Severance Hospital, Research Institute of Radiological Science, \\ Seoul; ${ }^{2}$ Severance Pediatric Liver Disease Research Group, Departments of ${ }^{3}$ Pediatric Surgery \\ and ${ }^{4}$ Surgery, ${ }^{5}$ Division of Gastroenterology, Hepatology and Nutrition, Department of \\ Pediatrics, ${ }^{6}$ Division of Maternal-Fetal Medicine, Department of Obstetrics and Gynecology, \\ Institute of Women's Life Medical Science, Yonsei University College of Medicine, Seoul, \\ Korea
}

Purpose: This study compared clinical and radiologic differences between cystic biliary atresia (CBA) and choledochal cyst (CC) type la/b.

Methods: Infants ( $\leq 12$ months old) who were diagnosed with cBA or CC type la/b from 2005 to 2019 were retrospectively reviewed. Imaging features on preoperative ultrasonography (US) and magnetic resonance imaging (MRI) were compared between the CBA and CC groups. Logistic regression and area under the receiver operating characteristic curve (AUC) analyses were performed for the diagnosis of CBA. Changes in cyst size were also evaluated when prenatal US exams were available.

Results: Ten patients (5.5\% of biliary atresia cases) with cBA (median age, 48 days) and 11 infants with CC type la/b (la:Ib=10:1; median age, 20 days) were included. Triangular cord thickness on US (cutoff, $4 \mathrm{~mm}$ ) showed 100\% sensitivity and 90.9\% specificity (AUC, 0.964; $95 \%$ confidence interval [CI], 0.779 to 1.000 ) and cyst size on MRI (cutoff, $2.2 \mathrm{~cm}$ ) had $70 \%$ sensitivity and $100 \%$ specificity (AUC, $0.900 ; 95 \% \mathrm{Cl}, 0.690$ to 0.987 ) for diagnosing CBA. Gallbladder mucosal irregularity on US and an invisible distal common bile duct on MRI were only seen in the CBA group (10 of 10). Only the CC group showed prenatal cysts exceeding $1 \mathrm{~cm}$ with postnatal enlargement.

Conclusion: Small cyst size $(<1 \mathrm{~cm})$ on prenatal US, triangular cord thickening $(\geq 4 \mathrm{~mm})$ and gallbladder mucosal irregularity on postnatal US, and small cyst size $(\leq 2.2 \mathrm{~cm})$ and an invisible distal common bile duct on MRI can discriminate CBA from CC type la/b in infancy.

Keywords: Biliary atresia; Choledochal cyst; Neonatal jaundice; Ultrasonography; Magnetic resonance imaging

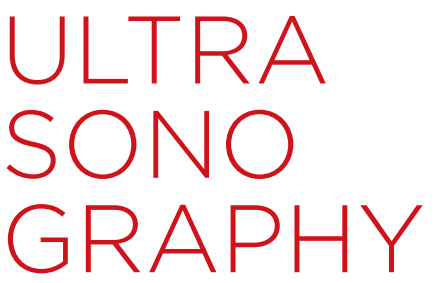

ORIGINAL ARTICLE

https://doi.org/10.14366/usg.20061 pISSN: 2288-5919 - eISSN: 2288-5943 Ultrasonography 2021;40:301-311

Received: April 28, 2020

Revised: July 15, 2020

Accepted: July 31, 2020

Correspondence to: Mi-Jung Lee, MD, PhD, Department of Radiology, Severance Hospital, Severance Pediatric Liver Disease Research Group, Research Institute of Radiological Science, Yonsei University College of Medicine, 50-1 Yonsei-ro, Seodaemun-gu, Seoul 03722, Korea

Tel. $+82-2-2228-7400$

Fax. +82-2-2227-8337

E-mail: mjl1213@yuhs.ac

This is an Open Access article distributed under the terms of the Creative Commons Attribution NonCommercial License (http://creativecommons.org/ licenses/by-nc/4.0/) which permits unrestricted noncommercial use, distribution, and reproduction in any medium, provided the original work is properly cited.

Copyright (C) 2021 Korean Society of Ultrasound in Medicine (KSUM)

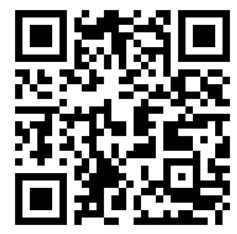

How to cite this article:

Shin HJ, Yoon H, Han SJ, Ihn K, Koh H, Kwon JY, et al. Key imaging features for differentiating cystic biliary atresia from choledochal cyst: prenatal ultrasonography and postnatal ultrasonography and MRI. Ultrasonography. 2021 Apr;40(2):301-311. 


\section{Introduction}

Biliary atresia (BA) is a disease involving sclerosing inflammation and obstruction of the bile ducts in early infancy that causes the liver to be unable to excrete bile into the duodenum $[1,2]$. BA is the most common cause of liver cirrhosis and hepatic failure in young children and often leads to liver transplantation [3]. However, the existence of multiple variants of $B A$ can make it difficult to diagnose $B A$. One such variant, cystic $B A(C B A)$, accounts for approximately $5 \%-10 \%$ of $B A$ cases $[4,5]$. When considering the three types of $B A$, cystic dilatation of the bile duct could be present at the point above or between the obliteration $[2,6,7]$. Therefore, a cyst of varying size could be present anywhere along the bile ducts, so this condition can mimic a choledochal cyst (CC) on imaging at the first presentation.

The diagnosis of BA early in infancy is important for the effective drainage of bile flow with the Kasai operation [8]. Early bile flow diversion before 60 postnatal days leads to better outcomes in $B A$ patients, even though $C B A$ cases were found to have higher native liver survival rates than patients with conventional $B A$ [7]. Moreover, the surgical methods differ between CBA and CC (hepaticojejunostomy and choledochojejunostomy, respectively) [9]. Therefore, early and accurate differentiation between CBA and CC is essential.

The typical sonographic findings for BA include a dysmorphic gallbladder (GB), an absent extrahepatic bile duct, a positive triangular cord sign, increased hepatic subcapsular flow, and an increased hepatic artery diameter [10-13]. In addition to ultrasonography (US), magnetic resonance imaging (MRI) and hepatobiliary scintigraphy can aid in the preoperative diagnosis of BA [14-16]. A microcyst in the porta hepatis is also known to be a specific sign for diagnosing BA [17]. However, distinguishing CBA from CC using imaging is particularly difficult when the cyst is large and extends into the extrahepatic space, resembling CC type la/ $\mathrm{b}$ according to the Todani classification [18]. Differentiation is also difficult in prenatal assessments $[19,20]$. Some authors suggested that $C B A$ and $C C$ could be interim entities belonging to the same pathologic continuum [21]. However, limited studies have focused on the differentiation of CBA with a large extrahepatic cyst from CC type la/b using various imaging modalities and prenatal images.

Therefore, the purpose of this study was to compare the clinical and radiologic differences between CBA and CC type la/b, including both prenatal and postnatal imaging.

\section{Materials and Methods}

\section{Subjects and Demographics}

The Institutional Review Board approved this retrospective study and waived informed consent. Infants ( $\leq 12$ months old) who were surgically confirmed to have CBA after the Kasai operation or CC type la or Ib from 2005 to 2019 were included and categorized into the CBA and CC groups, respectively. CBA was confirmed on intraoperative cholangiography and postoperative liver pathology. We only included cases of Todani classification type la/b (entire or segmental cystic dilatation of the extrahepatic bile duct) in the CC group because we surmised that the morphology of cysts in these types would resemble CBA. Other types, including type IV, were not included in the CC group because it was straightforward to exclude BA based on the presence and dilatation of intrahepatic bile ducts.

For the comparison of clinical and radiologic findings between CBA and CC groups, clinical findings such as age at the time of the postnatal US examination, sex, and initial preoperative laboratory results, including serum aspartate aminotransferase (AST), alanine transaminase (ALT), total bilirubin (T.bil), direct bilirubin (D.bil), $\gamma$ -glutamyl transferase ( $\mathrm{GTT}$ ), and alkaline phosphatase (ALP) were evaluated from a medical chart review. We also recorded the patients' age at the time of operation and the time interval between the postnatal US study and operation to evaluate whether any operations were delayed in these groups.

\section{Prenatal and Postnatal US}

Postnatal abdominal US was randomly performed with one of three machines (HDI 5000, Advanced Technology Laboratories, Bothell, WA, USA; iU22, Philips Healthcare, Bothell, WA, USA; and Aixplorer, SuperSonic Imagine, Aix-en-Provence, France) with both microconvex and linear probes of each machine throughout the study period. The thickness of the triangular cord (mm) and diameters of the hepatic arteries $(\mathrm{mm})$ were retrospectively reviewed by a pediatric radiologist with 10 years of experience in pediatric radiology. For GB, the presence of collapse and mucosal irregularity were evaluated. The size of cysts $(\mathrm{cm})$ and the presence of internal sludge in cysts were checked.

In the available prenatal US studies, the visibility and size of the $G B$ and changes in the cyst size (recorded using the longest diameter) from prenatal US to postnatal preoperative US were evaluated. The prenatal US examinations were performed by experienced gynecologists. The size of the GB (recorded using the longitudinal diameter) on prenatal and postnatal US was retrospectively measured by an experienced pediatric radiologist who was blinded to the patient's results. 


\section{Postnatal MRI}

We also reviewed preoperative abdominal MRI examinations, which were routinely performed at our hospital in patients who were suspected to have BA or CC. The MRI examinations were performed using a 1.5 T system (Intera Achieva, Philips Healthcare, Best, the Netherlands). The images were reviewed using T2-weighted fast spin-echo axial images (repetition time, 1,500-1,600 ms; echo time, $80 \mathrm{~ms}$; section thickness, $3 \mathrm{~mm}$; slice spacing, $3 \mathrm{~mm}$; flip angle, $90^{\circ}$; matrix size, $256 \times 256$; and field of view, $19-25 \mathrm{~cm}$ ), T1-weighted spin-echo axial images (repetition time, $525 \mathrm{~ms}$; echo time, $10 \mathrm{~ms}$; section thickness, $3 \mathrm{~mm}$; slice spacing, $3 \mathrm{~mm}$; flip angle, 90\%; number of excitations, 1; matrix size, 256×256; and field of view, 19-25 cm), and three-dimensional magnetic resonance cholangiopancreatography coronal images (repetition time, 1,500 ms; echo time, $650 \mathrm{~ms}$; section thickness, $2 \mathrm{~mm}$; slice spacing, $1 \mathrm{~mm}$; flip angle, 90\%; number of excitations, 1; matrix size, $256 \times 256$; and field of view, $19-25 \mathrm{~cm}$ ). On the MRI examinations, the same radiologist also reviewed the triangular cord thickness $(\mathrm{mm})$, diameters of the hepatic artery and portal vein $(\mathrm{mm}), \mathrm{GB}$ morphology (collapse, length, width, and ratio of $\mathrm{GB}$ length to width), and the longest diameter of the cyst (cm). In addition, the visualization of the distal common bile duct in the pancreas head, size of spleen $(\mathrm{cm})$, and the presence of splenomegaly based on the patients' age could be evaluated on MRI [22]. The thickness of the triangular cord sign on MRI was measured in accordance with a previous study [23].

All US and MRI examinations were performed after at least 4 hours of fasting for neonates and young infants.

\section{Statistical Analysis}

Statistical analyses were performed using the SPSS software package version 23 (IBM SPSS Statistics, IBM Corp., Armonk, NY, USA) and MedCalc version 19.1.3 (Ostend, Belgium). Values were presented as median values with ranges or as percentages within the corresponding group. Either the Mann-Whitney $U$ test or the Fisher exact test was used to compare the clinical and radiologic differences between the CBA and CC groups. The Wilcoxon signedrank test was used to compare measurements between US and MRI findings. Logistic regression and area under the receiver operating characteristic curve (AUC) analyses were performed for the diagnosis of $C B A$. A P-value of $<0.05$ was considered to indicate statistical significance.

\section{Results}

\section{Subjects and Laboratory Results}

Patients' demographic characteristics and imaging findings are summarized in Tables 1 and 2. Of the 182 patients with postoperatively confirmed BA during the study period, 10 (5.5\%; median, 48 days old; $M: F=2: 8)$ were diagnosed with $c B A$. During the study period, 11 (4.0\%; median, 20 days old; $M: F=3: 8$ ) of the 275 infants with CC had type la/b CC without intrahepatic biliary dilatation and were included in the CC group. In the CC group, 10 infants had type la CC and one had type Ib CC. Age and sex were not significantly different between the two groups $(P=0.605$ and $P>0.99$, respectively).

Among the laboratory results, AST, ALT, D.bil, and ALP levels were higher in the CBA group than in the CC group (Table 1). However, the T.bil and $\gamma \mathrm{GT}$ levels were not significantly different. The age at the time of operation was 14-105 days (median, 56 days) in the CBA group and 11-297 days (median, 25 days) in the CC group, which was not a significant difference $(P=0.654)$. The time interval

Table 1. Comparison of clinical findings between cBA and CC type la/b

\begin{tabular}{lccc}
\hline \multicolumn{1}{c}{ Variable } & CBA $(\mathrm{n}=10)$ & $\mathrm{CC}(\mathrm{n}=11)$ & P-value \\
\hline Age (day) & $48(2-95)$ & $20(1-185)$ & 0.605 \\
Sex (male:female) & $2: 08$ & $3: 08$ & $>0.99$ \\
Laboratory results & & & \\
AST (IU/L) & $146.5(27-288)$ & $35(14-140)$ & 0.008 \\
ALT (IU/L) & $110.5(17-264)$ & $21(7-123)$ & 0.020 \\
T.bil (mg/dL) & $9.9(5.1-9.7)$ & $7.6(0.2-14.7)$ & 0.152 \\
D.bil (mg/dL) & $7.8(3.5-11.1)$ & $1.0(0.1-3.5)$ & $<0.001$ \\
YGT (IU/L) & $430(233-775)$ & $273(9-1,213)$ & 0.131 \\
ALP $(I \mathrm{IU} / \mathrm{L})$ & $473(243-927)$ & $236(67-494)$ & 0.005 \\
\hline
\end{tabular}

Values are presented as median (range) or number.

$P$-values from the Mann-Whitney $U$ test or the Fisher exact test.

CBA, cystic biliary atresia; CC, choledochal cyst; AST, aspartate aminotransferase; ALT, alanine transaminase; T.bil, total bilirubin; D.bil, direct bilirubin; $\gamma$ GT, $Y$-glutamyl transferase; ALP, alkaline phosphatase. 
Table 2. Comparison of postnatal radiologic findings between cBA and CC type la/b

\begin{tabular}{|c|c|c|c|}
\hline Postnatal radiologic findings & $\mathrm{CBA}(\mathrm{n}=10)$ & $C C(n=11)$ & P-value \\
\hline \multicolumn{4}{|l|}{ US finding } \\
\hline Triangular cord (mm) & $5.2(4.2-6.9)$ & $3.1(1.9-5.0)$ & $<0.001$ \\
\hline HA diameter (mm) & $2.3(2.0-3.0)$ & $1.3(0.5-2.5)^{\mathrm{a})}$ & 0.007 \\
\hline Collapsed GB & $4 / 10(40.0)$ & 2/11 (18.2) & 0.361 \\
\hline GB mucosal irregularity & $10 / 10(100)$ & $0 / 11(0)$ & $<0.001$ \\
\hline Cyst size $(\mathrm{cm})$ & $2.0(0.7-3.4)$ & $3.4(2.0-6.1)$ & 0.002 \\
\hline Sludge in the cyst & $1 / 10(10.0)$ & $6 / 11(54.5)$ & 0.063 \\
\hline \multicolumn{4}{|l|}{ MRI finding } \\
\hline Triangular cord (mm) & $5.2(3.9-9.4)$ & $3.5(2.1-5.0)$ & $<0.001$ \\
\hline HA diameter (mm) & $2.8(2.3-3.6)$ & $2.5(1.2-3.0)$ & 0.029 \\
\hline PV diameter (mm) & $4.4(2.7-5.3)$ & $5.6(4.4-7.2)$ & 0.001 \\
\hline Collapsed GB & $4 / 10(40.0)$ & $1 / 11(9.1)$ & 0.149 \\
\hline GB length $(\mathrm{cm})$ & $3.2(0.9-5.8)$ & $2.9(2.2-4.2)$ & 0.809 \\
\hline GB width (cm) & $0.6(0.3-0.9)$ & $0.8(0.6-1.7)$ & 0.024 \\
\hline GB ratio & $4.7(2.8-7.3)$ & $3.7(2.5-4.7)$ & 0.016 \\
\hline Cyst size $(\mathrm{cm})$ & $2.1(0.6-4.3)$ & $3.6(2.7-7.2)$ & 0.001 \\
\hline Pancreas head bile duct visualization & $0 / 10(0)$ & $11 / 11(100)$ & $<0.001$ \\
\hline Spleen size $(\mathrm{cm})$ & $5.9(3.8-7.7)$ & $5.4(3.6-7.5)$ & 0.282 \\
\hline Splenomegaly & $2 / 10(20.0)$ & $1 / 11(9.1)$ & 0.586 \\
\hline
\end{tabular}

Values are presented as median (range) or number/total number (\%).

P-values from the Mann-Whitney $U$ test or the Fisher exact test.

CBA, cystic biliary atresia; CC, choledochal cyst; US, ultrasonography; HA, hepatic artery; GB, gallbladder; MRI, magnetic resonance imaging; PV, portal vein.

${ }^{a} n=$ The number of patients with hepatic diameter measurement was seven.

between the preoperative US study and operation was $6-12$ days (median, 6 days) in the CBA group and $5-112$ days (median, 8 days) in the CC group, which was also not a significant difference $(\mathrm{P}=0.387)$.

\section{Comparison of Prenatal and Postnatal US Findings between the CBA and CC Groups}

On postnatal US, the thickness of the triangular cord was greater in the CBA group than in the CC group (median, $5.2 \mathrm{~mm}$ vs. $3.1 \mathrm{~mm}$; $\mathrm{P}<0.001)$. The hepatic artery diameter was larger in the $C B A$ group than in the $\mathrm{CC}$ group (median, $2.3 \mathrm{~mm}$ vs. $1.3 \mathrm{~mm} ; \mathrm{P}=0.007$ ).

The GB was collapsed in $40.0 \%$ (4 of 10) of patients in the CBA group, whereas $18.2 \%$ (2 of 11) of the patients in the CC group had a collapsed $G B(P=0.361)$. $G B$ mucosal irregularity was observed in all $10(100 \%)$ patients in the CBA group, but was not detected in the $C C$ group $(P<0.001)$ (Fig. 1). The two cases of collapsed $G B$ in the CC group demonstrated a normal GB wall layer without a focal mucosal lesion. The GB length was $0.9-4.5 \mathrm{~cm}$ (median, $3.0 \mathrm{~cm}$ ) in the CBA group and 1.9-3.4 cm (median, $2.5 \mathrm{~cm}$ ) in the $\mathrm{CC}$ group $(\mathrm{P}=0.796)$. Two patients ( 2 of $11,18.2 \%$ ) in the $\mathrm{CBA}$ group and no patients in the $\mathrm{CC}$ group had a GB length less than $1.5 \mathrm{~cm}$.
The cyst size on postnatal US was $0.7-3.4 \mathrm{~cm}$ (median, $2.0 \mathrm{~cm}$ ) in the CBA group and $2.0-6.1 \mathrm{~cm}$ (median, $3.4 \mathrm{~cm}$ ) in the $\mathrm{CC}$ group. The cysts were significantly larger in the $\mathrm{CC}$ group than in the $\mathrm{CBA}$ group ( $P=0.002$ ) (Fig. 2). Sludge in the cyst was seen in only one patient (1 of 10,10.0\%) in the cBA group and in six patients (6 of $11,54.5 \%)$ in the CC group, which was not a significant difference ( $\mathrm{P}=0.063)$.

Only five patients at our institution (one in the CBA group and four in the CC group) had available prenatal US images. Three patients in the CC group had two serial prenatal US examinations. The GB was visible in three patients in the $C C$ group, with sizes of $1.3,1.9$, and $2.0 \mathrm{~cm}$. The $\mathrm{GB}$ was not detected in the $\mathrm{CBA}$ patient on prenatal US. The prenatal cyst size was $6 \mathrm{~mm}$ in the CBA group (Fig. 1A) and 18-46 mm in the CC group. When we compared the serial cyst size changes from the prenatal US and postnatal US and MRI images, as shown in Fig. 3, all of the patients in the CC group showed a gradual increase, with all cysts being larger than $1 \mathrm{~cm}$. In contrast, the size of the cyst in the patient in the CBA group was stable and less than $1 \mathrm{~cm}(6 \mathrm{~mm}$ on prenatal US and $7 \mathrm{~mm}$ on postnatal US and MRI). 


\section{MRI Findings}

All of the included patients underwent MRI. On MRI, the triangular cord was thicker in the CBA group than in the CC group (median, $5.2 \mathrm{~mm}$ vs. $3.5 \mathrm{~mm} ; \mathrm{P}<0.001$ ) (Table 2). The $c B A$ group had larger hepatic arteries and smaller portal vein diameters $(P=0.029$ and $P=0.001$, respectively). However, the prevalence of a collapsed $G B$ was not significantly different between the two groups ( $40 \%$ vs. 9.1\%, $P=0.149$ ). The $C B A$ group had narrower $G B s$ and larger $G B$ ratios, corresponding to an elongated shape (Fig. 1), than the CC group (Table 2). The cysts were larger in the CC group (median, 3.6 $\mathrm{cm}$; range, 2.7 to $7.2 \mathrm{~cm}$ ) than in the $\mathrm{CBA}$ group (median, $2.1 \mathrm{~cm}$; range, 0.6 to $4.3 \mathrm{~cm})(\mathrm{P}=0.001)$.

The difference between the postnatal US and MRI measurements was not significant for triangular cord thickness $(P=0.411)$, but was significant for the hepatic artery diameter (median, $0.5 \mathrm{~mm}$ overall, $0.4 \mathrm{~mm}$ in the CBA group, and $0.6 \mathrm{~mm}$ in $\mathrm{CC}$ group; $\mathrm{P}=0.003$ ) and cyst size (median, $2 \mathrm{~mm}$ overall, $0.5 \mathrm{~mm}$ in the cBA group, and 8 $\mathrm{mm}$ in the $\mathrm{CC}$ group; $\mathrm{P}=0.048$ ). However, the difference in these measurements was not significant between the two groups $(P=0.918$ for triangular cord thickness, $\mathrm{P}=0.193$ for the hepatic artery

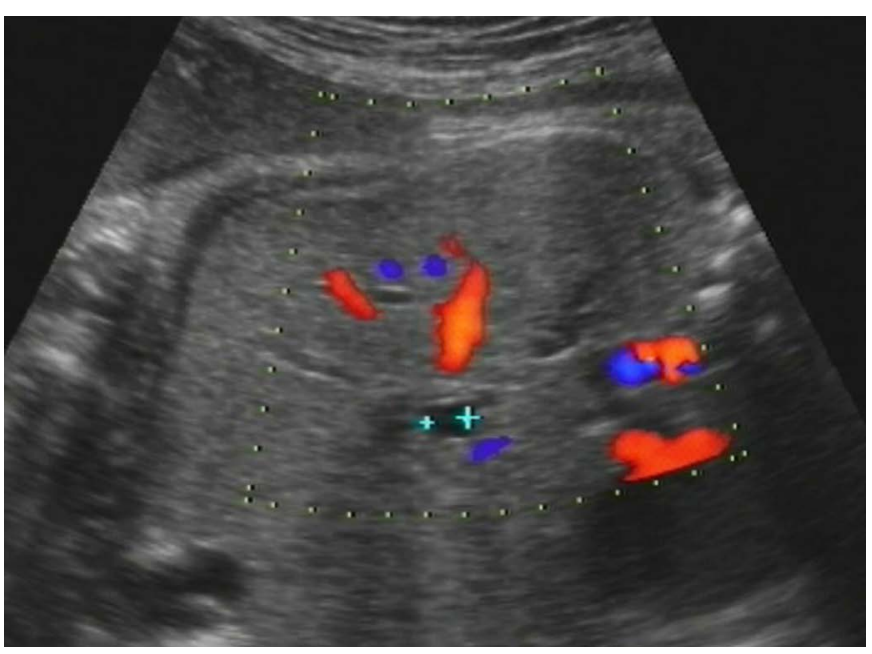

A

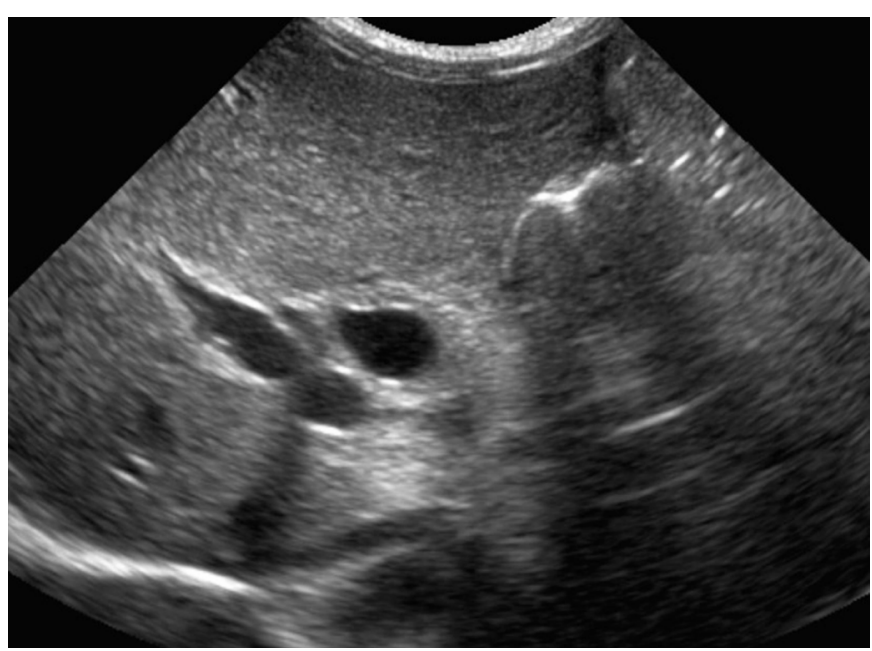

B

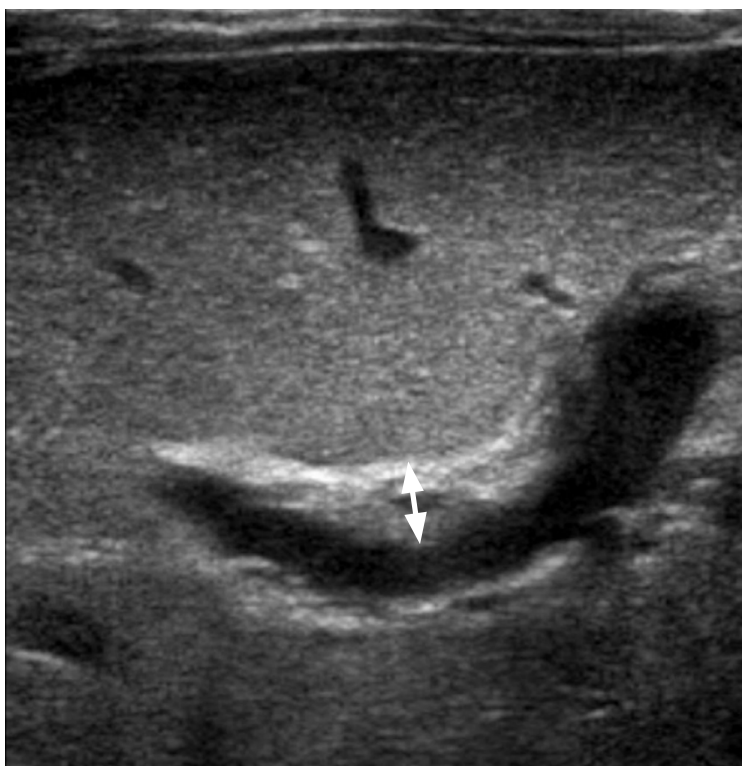

C

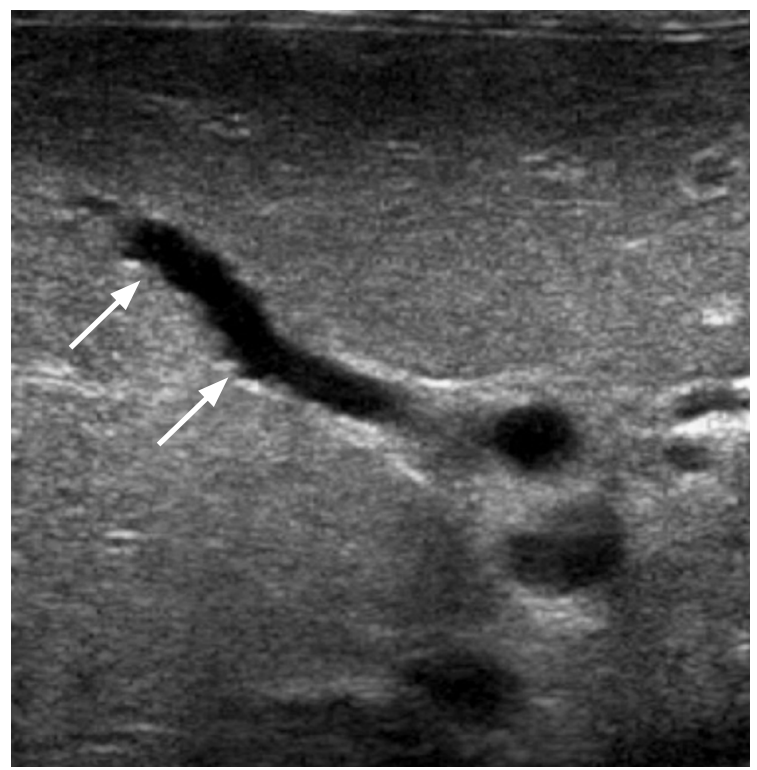

D

Fig. 1. A girl with cystic biliary atresia.

A. Prenatal ultrasonography (US) at a gestational age of 35 weeks shows a $0.6-\mathrm{cm}$ cyst around the hepatic hilum. B-D. Abdominal US on the second day after birth shows the cyst $(0.7 \mathrm{~cm})$ at the hepatic hilum $(B)$, increased periportal echo with triangular cord thickness (double arrow) of $4.8 \mathrm{~mm}(\mathrm{C})$, and mucosal irregularity (arrows) of the elongated gallbladder (GB) (D). 


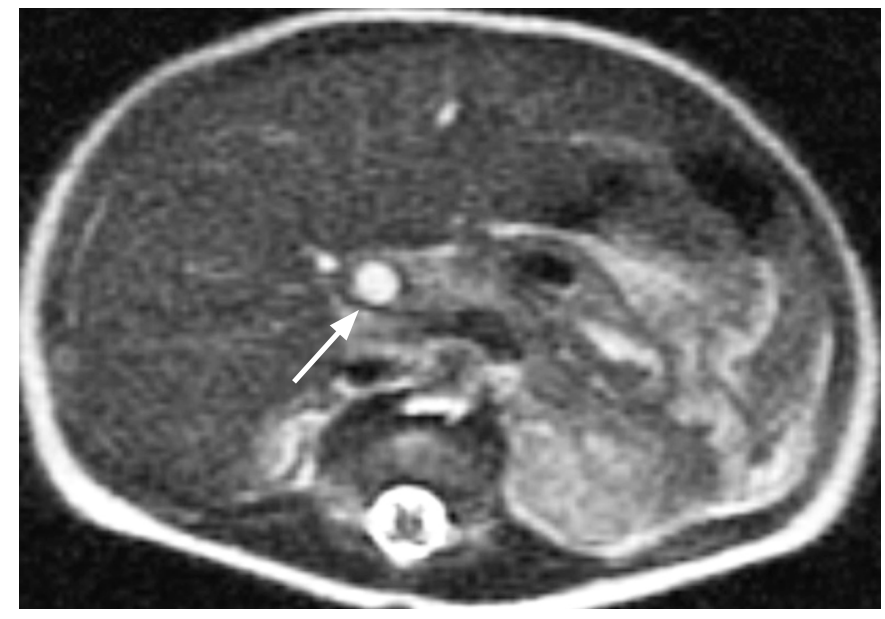

E

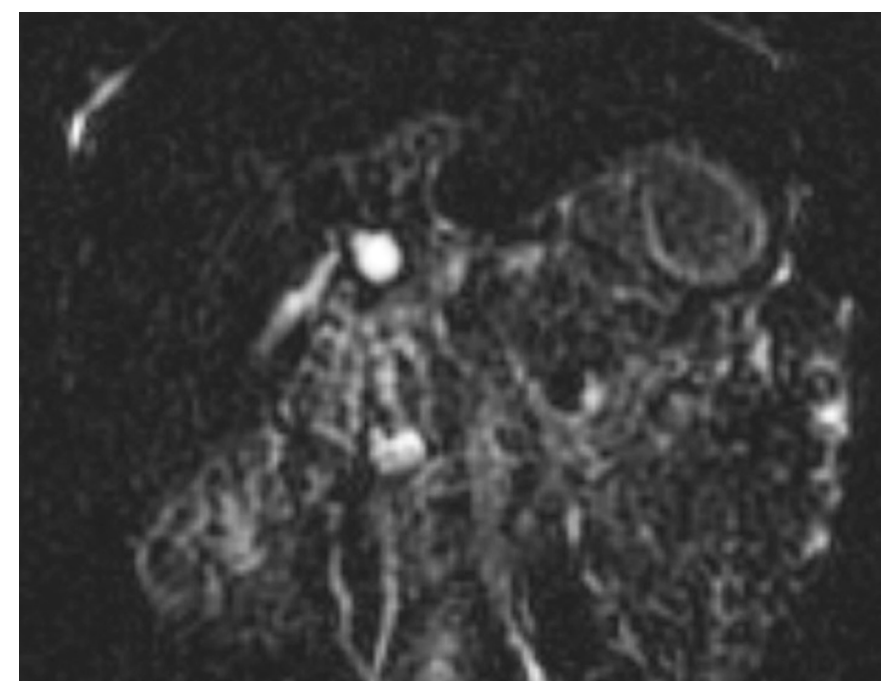

G

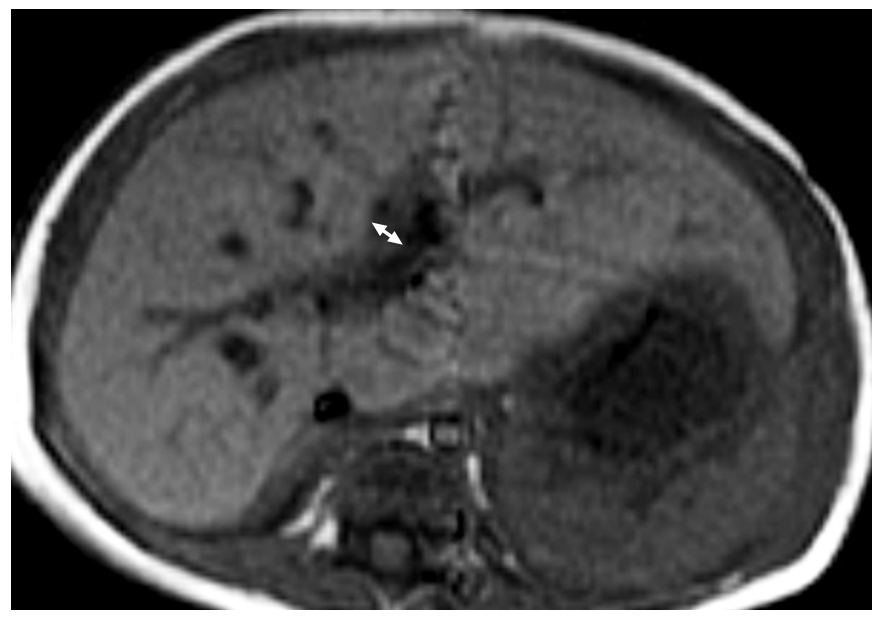

F

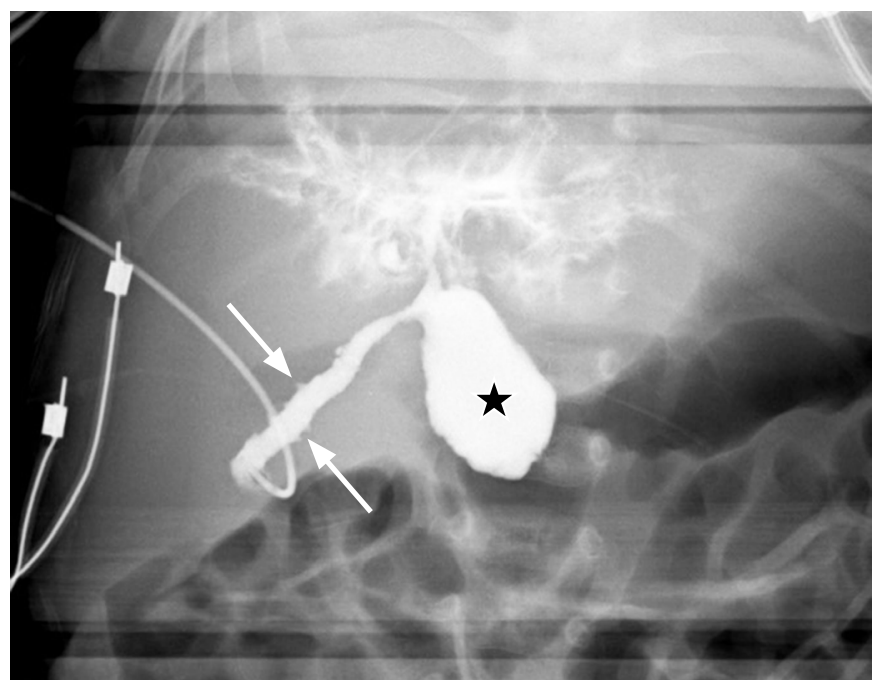

$\mathrm{H}$

Fig. 1. E-G. Preoperative abdominal magnetic resonance imaging also demonstrates a hepatic hilar cyst $(0.7 \mathrm{~cm}$, arrow) on a T2-weighted axial image (E), periportal thickening with a triangular cord thickness (double arrow) of $4.0 \mathrm{~mm}$ on a T1-weighted axial image (F), and no visible distal common bile duct (CBD) on three-dimensional magnetic resonance cholangiopancreatography $(\mathrm{G})$. $\mathrm{H}$. Intraoperative cholangiography confirmed an elongated GB with mucosal irregularity (arrows) connected with the cystic lesion (star) in the proximal CBD, an invisible distal CBD, and contrast leakage at the hepatic hilar area without visible normal bile duct branches. She was confirmed to have cystic biliary atresia and underwent the Kasai operation.

diameter, and $\mathrm{P}=0.349$ for cyst size).

On MRI, it was also possible to evaluate the visibility of the distal common bile duct and the presence of splenomegaly. The distal common bile duct was visualized in all patients in the CC group, while no patient had a visible distal common bile duct in the CBA group $(P<0.001)$. Spleen size and the prevalence of splenomegaly were not significantly different between the two groups (Table 2).

\section{Regression Analyses and Diagnostic Performance}

Univariate logistic regression analyses using the variables of triangular cord thickness and cyst size showed significant differences between the CBA and CC groups on both US and MRI. The odds ratio (OR) of triangular cord thickness on US for CBA was 26.015 (95\% confidence interval $[\mathrm{Cl}], 1.566$ to $432.164 ; \mathrm{P}=0.023$ ) and the OR of cyst size on US for CBA was $0.149(95 \% \mathrm{Cl}, 0.030$ to 0.748 ; $\mathrm{P}=0.021)$. On $M R I$, the $O R$ of triangular cord thickness for $C B A$ was $10.542(95 \% \mathrm{Cl}, 1.271$ to $87.400 ; P=0.029)$ and the OR of cyst size for CBA was $0.177(95 \% \mathrm{Cl}, 0.037$ to $0.860 ; \mathrm{P}=0.032)$.

For the diagnosis of $C B A$ in our study population, the AUC was $0.964(95 \% \mathrm{Cl}, 0.779$ to 1.000$)$ for US triangular cord thickness, $0.886(95 \% \mathrm{Cl}, 0.673$ to 0.982$)$ for US cyst size, $0.936(95 \% \mathrm{Cl}$, 0.739 to 0.996$)$ for MRI triangular cord thickness, and $0.900(95 \%$ 


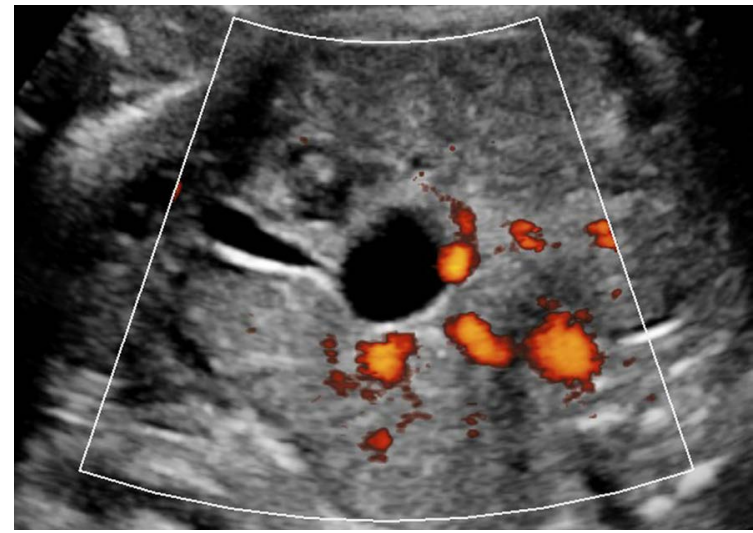

A

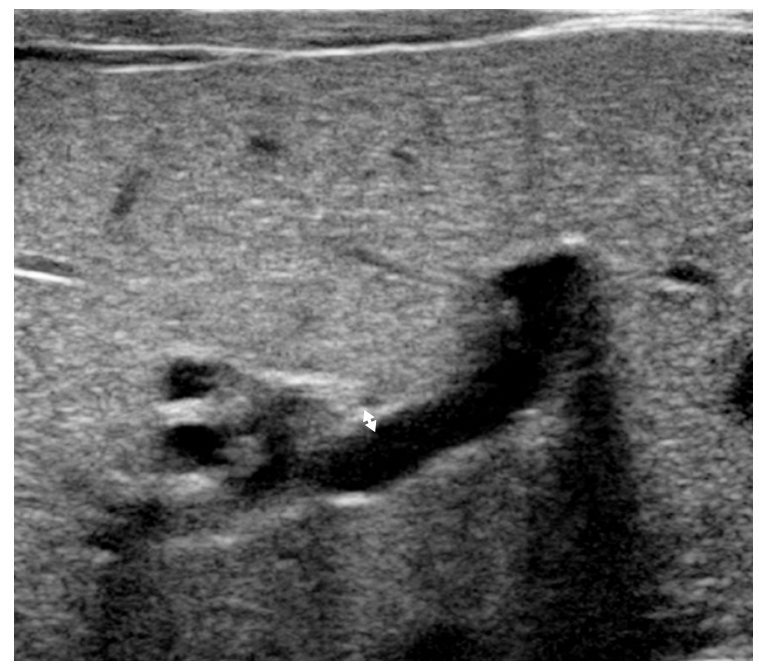

C

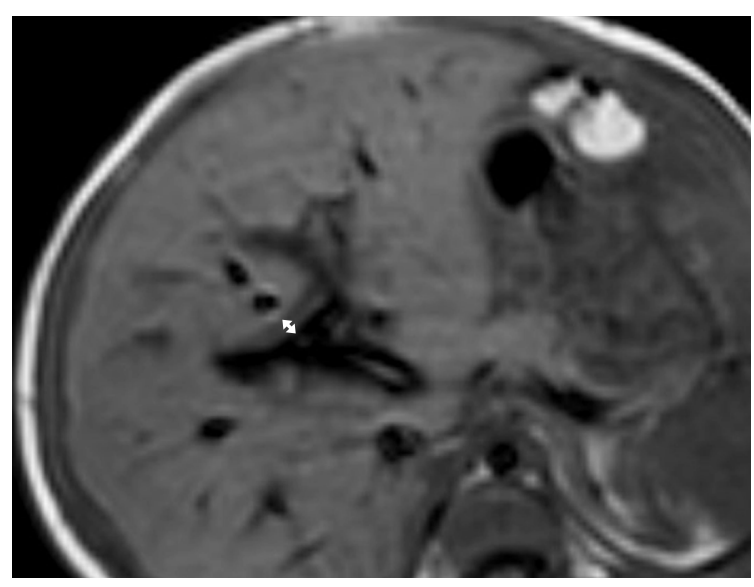

$\mathrm{E}$

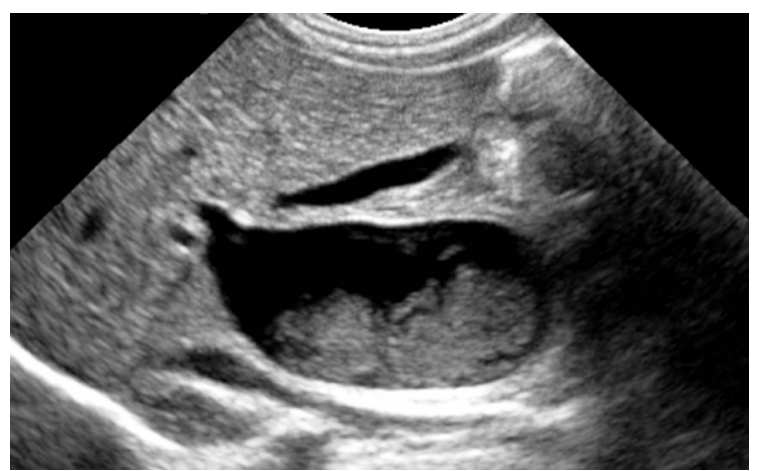

B

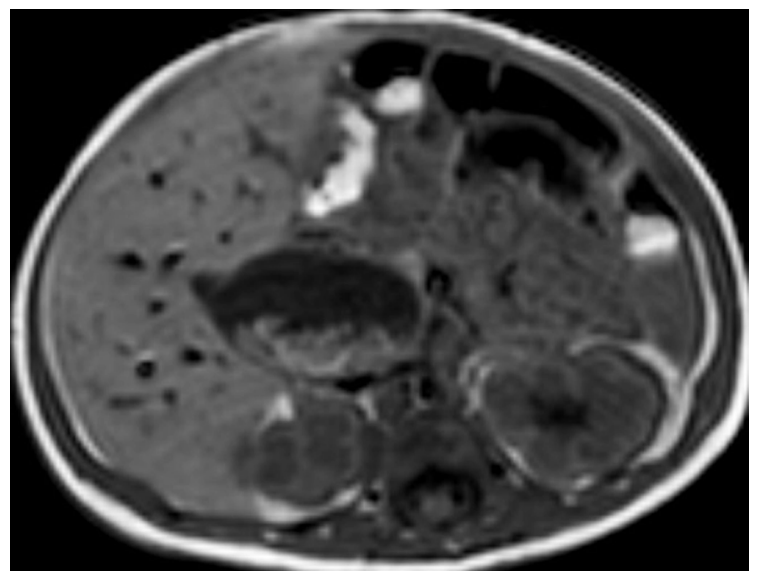

D

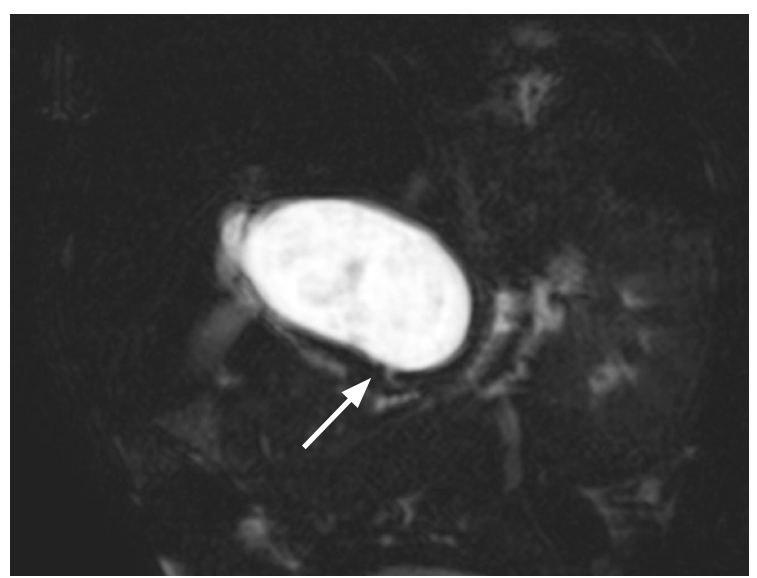

$\mathrm{F}$

Fig. 2. A boy with choledochal cyst type la.

A. Prenatal ultrasonography (US) at a gestational age of 31 weeks shows a hepatic hilar cystic lesion $(2.0 \mathrm{~cm})$ and gallbladder (GB). B, C. Postnatal abdominal US also shows the cystic lesion (about $2.6 \mathrm{~cm}$ ) at the hepatic hilar area with internal sludge, a grossly normal GB (B), and no remarkable periportal echo with a triangular cord thickness (double arrow) of $1.9 \mathrm{~mm}$ (C). D, E. T1-weighted axial magnetic resonance images demonstrates the same findings of the cyst $(3.4 \mathrm{~cm})$ with internal sludge (D) and periportal signal change with a triangular cord thickness (double arrow) of $3.9 \mathrm{~mm}$ (E). F. Magnetic resonance cholangiopancreatography shows patent distal common bile duct (arrow). The cystic lesion was confirmed as a choledochal cyst based on pathology findings. 
$\mathrm{Cl}, 0.690$ to 0.987 ) for MRI cyst size (all, $\mathrm{P}<0.001$ ) (Table 3). The AUCs of these four variables were not significantly different (Fig. 4).

The cutoff value of $\geq 4 \mathrm{~mm}$ for US triangular cord thickness showed $100 \%$ sensitivity and $90.9 \%$ specificity, while the cutoff value of $\geq 4 \mathrm{~mm}$ for MRI triangular cord thickness showed $90 \%$ sensitivity and $81.8 \%$ specificity. When considering cyst size, the cutoff value of $\leq 2.8 \mathrm{~cm}$ on US had $80 \%$ sensitivity and $81.8 \%$ specificity, while the cutoff value of $\leq 2.2 \mathrm{~cm}$ on MRI had $70 \%$ sensitivity and $100 \%$ specificity.

The combination of triangular cord thickness or cyst size on US with GB mucosal irregularity increased the AUC to 1.000. The combined diagnosis of triangular cord thickness or cyst size on MRI with a nonvisible distal common bile duct also increased the AUC to 1.000 (Table 3).

\section{Discussion}

CBA can be misdiagnosed as CC, especially when the cyst is large and not limited to the periportal tract. However, it is important to differentiate these two disease entities early in infancy because they have different clinical outcomes without an early intervention [24]. Our study showed that an increased triangular cord thickness (cutoff value of $4 \mathrm{~mm}$ on US, the same cutoff as in the original research on BA [10]), smaller cyst size, GB mucosal irregularity, and non-visibility

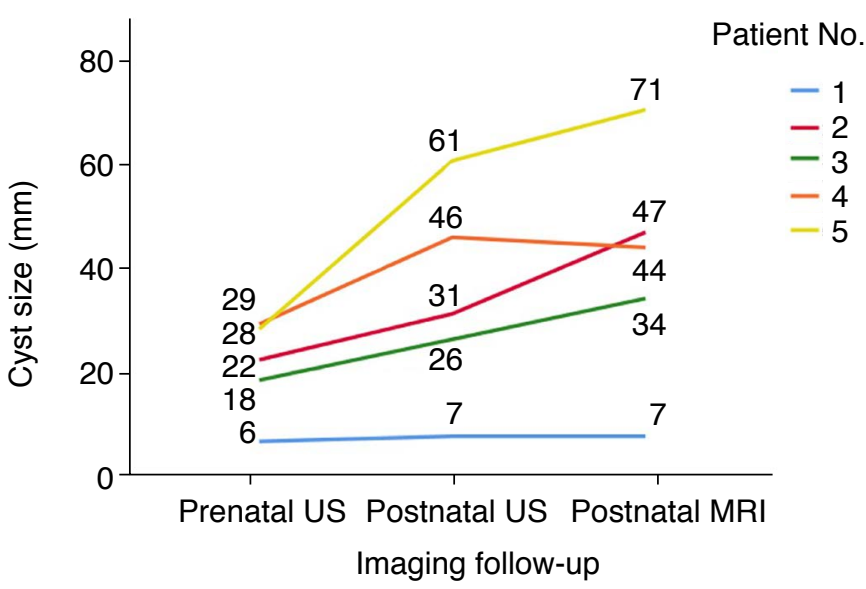

Fig. 3. Changes in cyst size from prenatal to postnatal imaging studies. This spaghetti plot demonstrates changes in cyst size from initial prenatal ultrasonography (US), postnatal preoperative US, and magnetic resonance imaging (MRI) in the order of the routine clinical examinations. The numbers in the graph denote the cyst size. Patient number 1 was a case of cystic biliary atresia and the other four patients had choledochal cyst type la. The cyst size was smaller than $1 \mathrm{~cm}$ and stable during follow-up in the cystic biliary atresia case, but was larger than $1 \mathrm{~cm}$ and showed a growth trend in all cases of choledochal cyst. of the distal bile duct on US or MRI were the key diagnostic features for differentiating CBA from CC type la/b, with excellent diagnostic performance, even though T.bil and $\gamma \mathrm{GT}$ levels were not significantly different between the two groups. These imaging features should be carefully examined when encountering infants with extrahepatic biliary dilatation.

In a previous study, the most frequently described difference between CBA and CC was the cyst size. Several studies mentioned that cysts were significantly smaller on postnatal US in CBA than in $C C$, with cutoff values ranging from 1.5 to $3.5 \mathrm{~cm}[5,24-26]$. Even though the cyst size in $C B A$ can be variable, and cysts can extend below the hepatic hilum, the cysts in infants with CBA were also significantly smaller than those in infants with CC in our study. In addition, on prenatal studies, the cysts in the $\mathrm{CC}$ group were all larger than $1 \mathrm{~cm}$ and showed a tendency for gradual growth after birth, while the cyst in the CBA group for which information was available was smaller than $1 \mathrm{~cm}$ and stable in size. Other studies also mentioned that the cyst size in infants with CBA was stable during follow-up in the prenatal period $[20,27,28]$ and smaller than that reported in a postnatal study [4], even though the size itself varied considerably across infants $(0.5-4.0 \mathrm{~cm})$. Obliteration between the intrahepatic and extrahepatic bile ducts in $B A$, rather

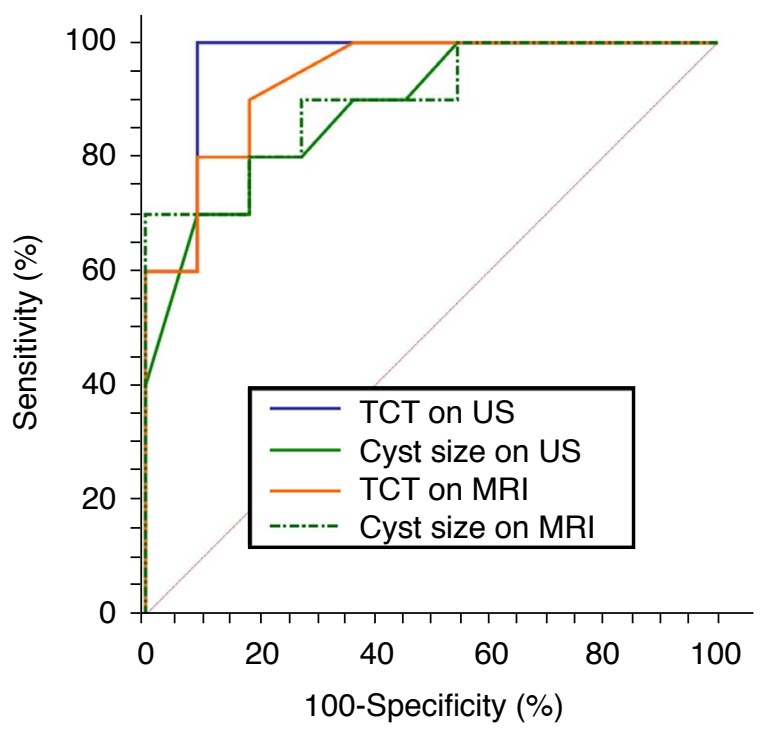

Fig. 4. Comparison of diagnostic performance between ultrasonography (US) and magnetic resonance imaging (MRI) parameters. Receiver operating characteristic curves of triangular cord thickness (TCT) and cyst size on US and MRI show similar areas under the curve (AUC). The AUC is 0.964 ( $95 \%$ confidence interval [CI], 0.779 to 1.000$)$ for US TCT, $0.886(95 \% \mathrm{Cl}, 0.673$ to 0.982$)$ for US cyst size, $0.936(95 \% \mathrm{Cl}, 0.739$ to 0.996$)$ for MRI TCT, and 0.900 $(95 \% \mathrm{Cl}, 0.690$ to 0.987$)$ for MRI cyst size, without statistically significant difference $(P>0.05)$. 
Table 3. Diagnostic performance of imaging findings in cystic biliary atresia

\begin{tabular}{|c|c|c|c|c|c|}
\hline & Cutoff value & AUC & 95\% Confidence interval & Sensitivity (\%) & Specificity (\%) \\
\hline \multicolumn{6}{|l|}{ Single parameter } \\
\hline \multicolumn{6}{|l|}{ US } \\
\hline Cyst size & $\leq 2.8 \mathrm{~cm}$ & 0.886 & $0.673-0.982$ & 80 & 81.8 \\
\hline GB mucosal irregularity & & & 100 & 100 & \\
\hline $\mathrm{TCT}$ & $\geq 4 \mathrm{~mm}$ & 0.936 & $0.739-0.996$ & 90 & 81.8 \\
\hline Cyst size & $\leq 2.2 \mathrm{~cm}$ & 0.900 & $0.690-0.987$ & 70 & 100 \\
\hline Invisible CBD & & & & 100 & 100 \\
\hline \multicolumn{6}{|l|}{ Combined parameters } \\
\hline \multicolumn{6}{|l|}{ US } \\
\hline TCT+invisible CBD & - & 1.000 & - & - & - \\
\hline Cyst size+invisible CBD & - & 1.000 & - & - & - \\
\hline
\end{tabular}

AUC, area under the curve; US, ultrasonography; TCT, triangular cord thickness; GB, gall bladder; MRI, magnetic resonance imaging; CBD, common bile duct.

than the normal development of the bile ducts, could contribute to the stable cyst size in utero [29]. Another theory suggested that overexpression of CD56 at the portal area represented dysregulation of the cell or intercellular matrix interactions in ductal plate remodeling in prenatal CBA patients [30]. These immune and inflammatory responses could affect various features of the size and growth patterns of cysts in CBA and CC prenatally. The differences in patterns of changes in cyst sizes from the prenatal to postnatal period could assist in the early diagnosis of CBA and the provision of appropriate interventions.

Triangular cord thickness is the most useful US feature for diagnosing BA and MRI can show comparable diagnostic performance to US using triangular cord thickness $[10,14,23]$. The combination of a thickened triangular cord sign, abnormal GB morphology, and non-visibility of the common bile duct showed excellent diagnostic performance for differentiating BA using both US and MRI [23]. Our study showed that these features could also help in the differentiation of CBA from CC. Zhou et al. [19] suggested that a positive triangular cord sign (cutoff, $4 \mathrm{~mm}$ ), smaller cyst size without internal sludge, hepatic artery enlargement, and GB mucosal irregularity could represent CBA on US. Those results are comparable with the findings of our study, except for sludge in the cyst, because one CBA patient in our study had sludge in the cyst. Furthermore, our study is meaningful since no other study has demonstrated imaging differences between US and MRI, including prenatal US. Even though US has benefits due to the better spatial resolution of neonatal abdominal US compared with MRI, both US and MRI showed excellent diagnostic performance for diagnosing CBA based on the triangular cord sign and cyst size. In addition, MRI has certain benefits, including clear visualization of the distal bile duct and its usefulness for preoperatively assessing vessel and bile duct anomalies, including anomalous pancreaticobiliary ductal union. In our study, a combination of US and MRI features, including triangular cord thickness $\geq 4 \mathrm{~mm}$, cyst size $\leq 2.2-2.8 \mathrm{~cm}$, GB mucosal irregularity, and a nonvisible distal common bile duct, showed excellent diagnostic performance.

The morphology of GB plays an important role in both prenatal and postnatal imaging. A small or nonvisible GB in the second trimester increases the suspicion of $B A$ on prenatal US $[28,29]$. Postnatally, the GB also shows an atretic or abnormal shape in CBA patients $[19,25,26]$. In our study, the case of $C B A$ with prenatal US had a nonvisible $G B$, whereas three of the four $C C$ patients with available prenatal US showed normal GB. Postnatal US also showed $G B$ mucosal irregularity in all cases of CBA and no cases of CC. Even though the $G B$ size was not significantly different between the CBA and CC groups in our study on either US and MRI, atretic GB $(<15 \mathrm{~mm})$ was only seen in the $C B A$ group. Moreover, abnormal GB morphology (a narrower GB and larger GB ratio) was a characteristic finding of $C B A$, rather than $C C$, on MRI. Not only an atretic or small $G B$, but also abnormal morphology (e.g., a tubular or elongated shape) can be an important marker for differentiating CBA from CC.

There are several limitations in this study. First, there was a small 
number of patients in each group because of the rarity of these diseases, even given the long study period at a tertiary hospital. We could not statistically compare the patterns of changing prenatal and postnatal cyst size because prenatal US images were only available for five patients, including one patient in the CBA group. Further research is needed on prenatal cyst size criteria. Second, because this was a retrospective study, there was a lack of information on some imaging features. A retrospective analysis of obtained images might identify differences in the hepatic artery diameter and cyst size depending on the imaging modality, even though the measurement difference was not significant between the groups. Third, neither pathologic correlations with the imaging findings nor clinical outcomes were assessed. Further evaluation through a multicenter study, including prenatal images, is needed for the clinical validation of these results.

Even though the cysts in CBA can resemble CC morphologically, key imaging features can aid in the differentiation of $C B A$ from $C C$ with excellent diagnostic performance. For $C B A$, a small cyst size $(<1 \mathrm{~cm})$ on prenatal US, triangular cord thickening $\geq 4 \mathrm{~mm}$, and GB mucosal irregularity on postnatal US, as well as a small cyst size $(\leq 2.2 \mathrm{~cm})$ and invisible distal common bile duct on MRI can discriminate CBA from CC type la/b in infancy. Careful evaluation and consideration of CBA are needed when we encounter a CC-like lesion in an infant.

ORCID: Hyun Joo Shin: https://orcid.org/0000-0002-7462-2609; Haesung Yoon: https://orcid.org/0000-0003-0581-8656; Seok Joo Han: https://orcid.org/0000-00015224-1437; Kyong Ihn: https://orcid.org/0000-0002-6161-0078; Hong Koh: https:// orcid.org/0000-0002-3660-7483; Ja-Young Kwon: https://orcid.org/0000-00033009-6325; Mi-Jung Lee: https://orcid.org/0000-0003-3244-9171

\section{Author Contributions}

Conceptualization: Shin HJ, Lee MJ. Data acquisition: Shin HJ, Yoon H, Han SJ, Ihn K, Kwon JY, Lee MJ. Data analysis or interpretation: Shin HJ, Yoon H, Lee MJ. Drafting of the manuscript: Shin HJ, Yoon H, Koh H, Lee MJ. Critical revision of the manuscript: Shin HJ, Lee MJ. Approval of the final version of the manuscript: all authors.

\section{Conflict of Interest}

No potential conflict of interest relevant to this article was reported.

\section{References}

1. Matsui A. Screening for biliary atresia. Pediatr Surg Int 2017;33:1305-1313.

2. Hartley JL, Davenport M, Kelly DA. Biliary atresia. Lancet 2009;374:1704-1713.
3. Kasahara M, Umeshita K, Sakamoto S, Fukuda A, Furukawa H, Uemoto $S$. Liver transplantation for biliary atresia: a systematic review. Pediatr Surg Int 2017;33:1289-1295.

4. Caponcelli E, Knisely AS, Davenport M. Cystic biliary atresia: an etiologic and prognostic subgroup. J Pediatr Surg 2008;43:16191624.

5. Suzuki T, Hashimoto T, Hussein MH, Hara F, Hibi M, Kato T. Biliary atresia type I cyst and choledochal cyst [corrected]: can we differentiate or not? J Hepatobiliary Pancreat Sci 2013;20:465-470.

6. Ohi R, Ibrahim M. Biliary atresia. Semin Pediatr Surg 1992;1:115124.

7. Ihn K, Ho IG, Lee JH, Na Y, Lee D, Han SJ. Comparison of the outcomes of biliary atresia with cystic degeneration and isolated biliary atresia: a matched-pair analysis. J Pediatr Surg 2020;55:2177-2182.

8. Hwang SM, Jeon TY, Yoo SY, Choe YH, Lee SK, Kim JH. Early US findings of biliary atresia in infants younger than 30 days. Eur Radiol 2018;28:1771-1777.

9. Song Z, Dong R, Shen Z, Chen G, Yang Y, Zheng S. Surgical outcome and etiologic heterogeneity of infants with biliary atresia who received Kasai operation less than 60 days after birth: A retrospective study. Medicine (Baltimore) 2017;96:e7267.

10. Lee HJ, Lee SM, Park WH, Choi SO. Objective criteria of triangular cord sign in biliary atresia on US scans. Radiology 2003;229:395400

11. Zhou LY, Wang W, Shan QY, Liu BX, Zheng YL, Xu ZF, et al Optimizing the US diagnosis of biliary atresia with a modified triangular cord thickness and gallbladder classification. Radiology 2015:277:181-191.

12. Lee MS, Kim MJ, Lee MJ, Yoon CS, Han SJ, Oh JT, et al. Biliary atresia: color doppler US findings in neonates and infants. Radiology 2009;252:282-289.

13. Kim WS, Cheon JE, Youn BJ, Yoo SY, Kim WY, Kim IO, et al. Hepatic arterial diameter measured with US: adjunct for US diagnosis of biliary atresia. Radiology 2007;245:549-555.

14. Kim MJ, Park YN, Han SJ, Yoon CS, Yoo HS, Hwang EH, et al. Biliary atresia in neonates and infants: triangular area of high signal intensity in the porta hepatis at T2-weighted MR cholangiography with US and histopathologic correlation. Radiology 2000;215:395401.

15. Fitoz S, Erden A, Boruban S. Magnetic resonance cholangiopancreatography of biliary system abnormalities in children. Clin Imaging 2007;31:93-101.

16. Kianifar HR, Tehranian S, Shojaei P, Adinehpoor Z, Sadeghi R, Kakhki $V R$, et al. Accuracy of hepatobiliary scintigraphy for differentiation of neonatal hepatitis from biliary atresia: systematic review and meta-analysis of the literature. Pediatr Radiol 2013;43:905-919.

17. Koob M, Pariente D, Habes D, Ducot B, Adamsbaum C, FranchiAbella $\mathrm{S}$. The porta hepatis microcyst: an additional sonographic 
sign for the diagnosis of biliary atresia. Eur Radiol 2017;27:18121821.

18. Lewis VA, Adam SZ, Nikolaidis P, Wood C, Wu JG, Yaghmai V, et al. Imaging of choledochal cysts. Abdom Imaging 2015;40:15671580.

19. Zhou LY, Guan BY, Li L, Xu ZF, Dai CP, Wang W, et al. Objective differential characteristics of cystic biliary atresia and choledochal cysts in neonates and young infants: sonographic findings. J Ultrasound Med 2012;31:833-841.

20. Hasegawa T, Sasaki T, Kimura T, Sawai T, Nose K, Kamata S, et al. Prenatal ultrasonographic appearance of type IIId (uncorrectable type with cystic dilatation) biliary atresia. Pediatr Surg Int 2002;18:425-428.

21. Mahalik SK, Mitra S, Patra S, Das K. Cystic biliary atresia or atretic choledochal cyst: a continuum in infantile obstructive cholangiopathy. Fetal Pediatr Pathol 2019;38:477-483.

22. Megremis SD, Vlachonikolis IG, Tsilimigaki AM. Spleen length in childhood with US: normal values based on age, sex, and somatometric parameters. Radiology 2004;231:129-134.

23. Kim YH, Kim MJ, Shin HJ, Yoon H, Han SJ, Koh H, et al. MRI-based decision tree model for diagnosis of biliary atresia. Eur Radiol 2018;28:3422-3431.
24. Tang J, Zhang D, Liu W, Zeng JX, Yu JK, Gao Y. Differentiation between cystic biliary atresia and choledochal cyst: a retrospective analysis. J Paediatr Child Health 2018;54:383-389.

25. Huang FC, Hwang KP. Differential diagnosis of infantile choledochal cyst with or without biliary atresia. Acta Paediatr Taiwan 2006;47:175-180.

26. Kim WS, Kim IO, Yeon KM, Park KW, Seo JK, Kim CJ. Choledochal cyst with or without biliary atresia in neonates and young infants: US differentiation. Radiology 1998;209:465-469.

27. Redkar R, Davenport M, Howard ER. Antenatal diagnosis of congenital anomalies of the biliary tract. J Pediatr Surg 1998;33:700-704.

28. Koukoura O, Kelesidou V, Delianidou M, Athanasiadis A, Dagklis T. Prenatal sonographic diagnosis of biliary tract malformations. J Clin Ultrasound 2019;47:292-297.

29. Shen $O$, Sela HY, Nagar H, Rabinowitz R, Jacobovich E, Chen D, et al. Prenatal diagnosis of biliary atresia: a case series. Early Hum Dev 2017;111:16-19.

30. Okada T, Itoh T, Sasaki F, Cho K, Honda S, Todo S. Comparison between prenatally diagnosed choledochal cyst and type-1 cystic biliary atresia by CD56-immunostaining using liver biopsy specimens. Eur J Pediatr Surg 2007;17:6-11. 